m/s

médecine/sciences $1999 ; 15: 139-40$

\title{
ENDOCRINOLOGIE DE LA REPRODUCTION : L'OVAIRE DANS TOUS SES ÉTATS
}

\section{Philippe Bouchard}

\author{
ADRESSE \\ Philippe Bouchard : docteur en médecine, profes- \\ seur des universités, praticien hospitalier. Service \\ d'endocrinologie, Hôpital Saint-Antoine, $\mathrm{Pa}-$ \\ villon de l'Horloge, 184, rue du Faubourg- \\ Saint-Antoine, 75571 Paris Cedex 12, France.
}

'endocrinologie de la reproduction concerne beaucoup de domaines de la vie et de la médecine. Elle s'intéresse notamment au vieillissement, aux conséquences de la ménopause, à l'infertilité et à la contraception. Les besoins mondiaux pour ces approches sont considérables puisque ce domaine s'intéresse à la fertilité, au contrôle des naissances, à la qualité de vie des femmes (ce que les Anglo-Saxons appellent Reproductive Health), et au caractère harmonieux du vieillissement. Il s'agit donc d'une médecine à la fois à la pointe de la technologie, comme les techniques de procréations médicalement assistées ou de diagnostic préimplantatoire, mais aussi une médecine au cœur des dialogues Nord-Sud, puisque la plus grande demande, en particulier de contraception, vient des pays en voie de développement et que, justement, les pouvoirs publics de ces pays n'ont guère les moyens de proposer ces traitements. En outre, la recherche biotechnologique et pharmaceutique, très active dans ce domaine, n'est pas très motivée pour produire des produits efficaces, sans risque et à moindre coût.

Sous nos climats, il est habituellement considéré que la médecine et l'endocrinologie de la reproduction ont atteint un niveau de développement conséquent et que le financement de cette recherche n'est plus prioritaire. Ce numéro de médecine/ sciences est là pour prouver le contraire. Les progrès faits dans ce domaine au cours des 10 dernières années ont été fulgurants et, à la lecture de ce numéro, le lecteur comprendra aisé- ment qu'il s'agit d'une aventure transversale dans laquelle les médecins et les chercheurs de tous domaines - de la génétique à la biologie cellulaire, à l'endocrinologie fondamentale et à la médecine clinique - ont concouru à part égale. Ainsi, le contrôle du phénomène ovarien dominant qu'est l'atrésie folliculaire [1], et la reconnaissance des gènes qui la règlent, sont-ils d'un intérêt thérapeutique majeur pour repousser ou prévoir la ménopause [2]. Les relations reproduction-nutrition sont, elles aussi, au premier plan grâce à l'étude du mécanisme d'action de la leptine [3]. Ainsi, la physiologie d'une affection aussi fréquente qu'est l'aménorrhée dite hypothalamique, d'origine nutritionnelle pour l'essentiel, source de carence œestrogénique et de graves conséquences médicales, va-t-elle s'éclairer et conduire à une approche plus rationnelle de ces patientes [4]. Deux autres conséquences exceptionnelles des travaux présentés dans ce numéro sont sans conteste l'analyse du mécanisme de l'effet vasculaire des œestrogènes [5] et du contrôle de la croissance folliculaire [6-8]. L'effet vasculaire des œestrogènes est d'un intérêt capital pour justifer le traitement de la ménopause. Si l'effet osseux de celui-ci est raisonnablement certain, en tout cas jugé sur la densité osseuse, les effets vasculaires restent mal compris et leur impact clinique encore incertain. Les deux grandes études épidémiologiques en cours aux États-Unis et en GrandeBretagne viendront probablement en 2007 apporter des arguments décisifs, parallèlement aux données expérimentales présentées dans ce numé- 
ro. Enfin, la maturation ovocytaire in vitro à partir de prélèvement ovarien représente un grand espoir pour traiter certaines femmes à risque d'insuffisance ovarienne précoce ou à risque d'hyperstimulation $[9,10]$. Finalement, il n'est pas utopique de penser que la contraception bénéficiera grandement de ces nouvelles connaissances, ce qui permettra peutêtre d'utiliser une approche ovarienne directe du contrôle de la fertilité, n'altérant pas les sécrétions hormonales et sans impact général.

Je remercie tous les collègues et amis qui ont contribué à ce prestigieux numéro de médecine/sciences qui va, je l'espère, participer à la relance active de la recherche et au financement de celle-ci

\section{RÉFÉRENCES}

1. Monniaux D, Mandon-Pépin B, Monget P. L'atrésie folliculaire, un gaspillage programmé. Med Sci 1999 ; 15 : 157-66.

2. Vasseur C, Christin-Maître S, Bouchard P. Physiopathologie de l'insuffisance ovarienne prématurée : faits et perspectives. $\mathrm{Med} \mathrm{Sci}$ 1999 ; 15 : 204-11.

3. Bruneau G, Vaisse C, Caraty A, Monget P. La leptine : une clé pour la reproduction. Med Sci 1999 ; 15 : 191-6.

4. Bringer J, Lefebvre P, Renard E. Nutrition et physiologie ovarienne. Med Sci $1999 ; 15$ : 197-203.

5. Arnal J, Elhage R, Maret A, Rami J, Faye J, Bayard F. Estrogènes et athérosclérose : données récentes et perspectives. Med Sci $1999 ; 15: 212-8$.

6. Young J, Gougeon A, Schaison G. Le cycle ovarien. Med Sci 1999; $15: 183-90$.
7. Combarnous Y. Structure et relations structure-activité des FSH recombinantes humaines. Med Sci 1999 ; 15 : 167-74.

8. Misrahi M, Beau I, Atger M, et al. Les récepteurs des gonadotrophines. Med Sci $1999 ; 15: 175-82$.

9. Monget P, Hembert S, Binart N, Gougeon A, Panthier J. La physiologie ovarienne : ce que nous disent les souris... Med Sci 1999 ; $15: 141-7$.

10. Mermillod P, Marchal R. La maturation de l'ovocyte de mammifères. Med Sci 1999 ; $15: 148-56$

\section{TIRÉS À PART}

$\mathrm{Ph}$. Bouchard.

\section{I/S}

